\title{
A new in vivo analysis model to detect sexually dimorphic rat liver cytochrome P450 gene expression dependent on growth hormone secretory patterns
}

\author{
Motoyo MARUYAMA ${ }^{1)}$, Masahiko FUJISAWA ${ }^{2)}$, Makoto YOKOSUKA ${ }^{3)}$, Toru R. SAITO ${ }^{3)}$, \\ Shin-ichi HAYAMA ${ }^{4)}$, Toshio AKIMOTO ${ }^{1)}$, and Yoji HAKAMATA ${ }^{2)}$ \\ 1)Divison of Laboratory Animal Science, Nippon Medical School, 1-1-5, Sendagi, Bunkyo-ku, Tokyo 113-8602, \\ Japan \\ ${ }^{2)}$ Department of Basic Science, School of Veterinary Nursing and Technology, Faculty of Veterinary Science, Nippon \\ Veterinary and Life Science University, 1-7-1, Kyonancho, Musashino, Tokyo 180-8602, Japan \\ ${ }^{3)}$ Laboratory of Comparative and Behavioral Medicine, School of Veterinary Medicine, Faculty of Veterinary \\ Science, Nippon Veterinary and Life Science University, 1-7-1, Kyonancho, Musashino, Tokyo 180-8602, Japan \\ 4) Laboratory of Wildlife Medicine, School of Veterinary Medicine, Faculty of Veterinary Science, Nippon Veterinary \\ and Life Science University, 1-7-1, Kyonancho, Musashino, Tokyo 180-8602, Japan
}

\begin{abstract}
Several drug-metabolizing cytochrome P450 (CYP) enzymes exhibit sexual dimorphism depending on the pituitary growth hormone $(\mathrm{GH})$ secretory patterns. However, the mechanism underlying CYP sexual dimorphism remains unclear. We previously established a transgenic (AlbDsRed2 Tg) rat that expressed red fluorescent DsRed2 protein, particularly in hepatocytes, to visualize cell differentiation and multiplication and found that hepatic DsRed2 expression exhibited sexual dimorphism that was limited to adult males. In this study, we compared the expression patterns between sexual dimorphic Cyps and DsRed2 in Tg rats after experimentally reversing the GH secretory patterns in males and females. Postnatal day 1 male and female Tg rats were gonadectomized and then testosterone propionate $(0.25 \mathrm{mg} / \mathrm{rat})$ was subcutaneously administered to ovariectomized females immediately after surgery. Cyp mRNA and DsRed2 expression levels were quantified using RT-PCR and an in vivo imaging system, respectively. GH-dependent Cyps and hepatic DsRed2 expression patterns were reversed in males and females at 9 weeks after birth and were significantly correlated $(P<0.05)$. This suggested that DsRed2 expression in these $\mathrm{Tg}$ rats depended on $\mathrm{GH}$ secretory patterns. Based on DsRed2 fluorescence, this $\mathrm{Tg}$ rat model could become a tool to readily and effectively evaluate changes in $\mathrm{GH}$-dependent Cyp expression.
\end{abstract}

Key words: cytochrome P450, in vivo imaging, sexual dimorphism, transgenic rat

\section{Introduction}

The drug-metabolizing cytochrome P450 enzymes (CYPs) facilitate steroid and xenobiotic metabolism in the liver and are important for maintaining the body's homeostasis. CYPs are classified by specific amino acids, and more than 500 subspecies have been found in humans, with differences between males and females as well as between different animal species. Several CYP classes that reflect sexual dimorphism have been identi-

(Received 1 April 2016 / Accepted 31 May 2016 / Published online in J-STAGE 29 June 2016)

Adress corresponding: Y. Hakamata, Department of Basic Science, School of Veterinary Nursing and Technology, Faculty of Veterinary Science, Nippon Veterinary and Life Science University, 1-7-1, Kyonancho, Musashino, Tokyo 180-8602, Japan

(C)2016 Japanese Association for Laboratory Animal Science 
fied $[2,4,11,12,14,15,23]$. In rats, Cyp2c11, a malespecific isoform that comprises $50 \%$ of total $\mathrm{P} 450$ in the male liver, and Cyp2c12, a female-specific isoform that comprises $40 \%$ of total $\mathrm{P} 450$ in the female liver, are typical sexually dimorphic CYPs. In humans, women exhibit higher CYP3A4 expression than do men [2], and CYP3A4 is involved in metabolizing about $50 \%$ of commercial drugs [4].

These sex differences in CYPs result in clinically important problems because of the differences between men and women in the effectiveness and side effects of drugs. However, the molecular mechanisms underlying these sex differences in CYPs remain unclear. In studies with rodents, establishing CYP sexual dimorphism depended on growth hormone (GH) secretory patterns via the hypothalamus-pituitary-liver axis, which was determined by the sex steroid hormone environment during the perinatal period [18, 21]. CYP sexual dimorphisms were disrupted by altering serum GH levels [10].

Transgenic ( $\mathrm{Tg}$ ) animals are useful models for analyzing various gene functions in a living body. In particular, $\mathrm{Tg}$ rats provide considerable genomic information similar to that obtained with mice, although the larger body size of the rat makes it preferable for transplant and stem cell research. Fluorescent or luminescent proteins, such as green fluorescent protein (GFP), red fluorescent protein2 (DsRed2), or firefly luciferase, can be used to identify particular cells in the body. Thus, a number of $\mathrm{Tg}$ animals that harbor these marker proteins have provided tools for biomedical research $[6,13]$.

We have generated many useful $\mathrm{Tg}$ rat models for use in regenerative medicine research [5, 7], including one that specifically expressed DsRed2 in the liver [17]. AlbDsRed2 Tg rats express DsRed2, a red fluorescent protein derived from the genus Discosoma, particularly in hepatocytes under the control of a mouse albumin enhancer/promoter. Because DsRed2 fluorescence can be visualized only when using excitation light of a specific wavelength, it is a useful analytical tool for simply and noninvasively tracking the migration and differentiation of cells. Hepatic DsRed2 expression was detected only in adult male rats, which reflected sexual dimorphism [1]. In addition, the DsRed2 expression pattern did not change after performing gonadectomy in both male and female adult rats, although DsRed2 expression did appear in hypophysectomized females or in cultured female hepatocytes in the absence of pituitary hormones. This strongly suggested that the sexual dimorphism of
DeRed2 expression in these $\mathrm{Tg}$ rats was regulated by hypothalamic-pituitary hormones, including $\mathrm{GH}$.

In this study, to determine the relevance of GH-dependent endogenous Cyp expression and sexual dimorphic DsRed2 expression by Alb-DsRed2 Tg rats, we experimentally reversed the GH secretory patterns of male and female Alb-DsRed2 Tg rats. Using this model, we investigated the influence of GH secretory patterns on GH-dependent Cyp expression and hepatic DsRed2 expression in adult rats. We propose that liver-specific DsRed2 fluorescence in this $\mathrm{Tg}$ rat can be used to monitor any changes in GH-dependent Cyp expression that indicates a sexual dimorphism and can also become a tool to investigate the mechanisms underlying the establishment of a sexual dimorphism.

\section{Materials and Methods}

\section{Experimental animals}

For all experiments, we used Alb-DsRed2 Tg heterozygous male and female Wistar-Tg (Alb-DsRed2) 34Jmsk rats, which were established as reported previously [17]. These rats were maintained under controlled conditions, with temperature at $20-25^{\circ} \mathrm{C}$, humidity of $40 \%-70 \%$, and a $12 \mathrm{~h}$ light $/ 12 \mathrm{~h}$ dark cycle (lights on at 0700). Food and water were provided ad libitum. All experiments in this study were conducted in accordance with the Nippon Veterinary and Life Science University Guide for Laboratory Animals.

\section{Surgical procedures and experimental schedule}

On postnatal day 1, Alb-DsRed2 Tg heterozygous male and female rats underwent castration (Cast) or ovariectomy (OVX) while under isoflurane anesthesia (Intervet, Tokyo, Japan), and were designated the Cast male and OVX female groups, respectively. To reverse the GH secretory patterns in male and female Tg rats in adulthood, according to previous reports [9], testosterone propionate (TP) $(0.25 \mathrm{mg} /$ head in $0.05 \mathrm{ml}$ olive oil) was administered subcutaneously into selected ovariectomized females immediately after surgery, and these rats were designated the OVX $+\mathrm{TP}$ female group. Some female rats did not undergo ovariectomy but were injected with TP (TP female group) and used as controls. Some male and female rats did not undergo castration or ovariectomy (sham male and sham female groups).

Pups were housed with their mothers until postnatal day 20 and bred 9 weeks after birth. These rats were 
euthanized by intraperitoneal administration of pentobarbital $(65 \mathrm{mg} / \mathrm{kg})$ and left liver lobes were partially removed for analysis. These rats were subsequently perfused through the heart with heparinized saline (5 IU/ $\mathrm{ml}$ ), followed by a $10 \%$ formalin buffer solution. Brains were removed and post-fixed in the same solution and then transferred to $30 \%$ sucrose in $0.1 \mathrm{M}$ phosphate buffer until they settled.

\section{Quantifying hepatic DsRed2 expression}

At 9 weeks after birth, hepatic DsRed2 expression levels in each rat were quantified using an IVIS bioimaging system (Xenogen, Alameda, CA). DsRed2 fluorescence in liver tissue was excited with a light at $560 \mathrm{~nm}$, after which light emission $(600 \mathrm{~nm})$ and images were acquired using a cooled charge-coupled device camera. Living Image software (Xenogen, Alameda, CA) was used to quantify fluorescent signals, which were expressed as digitized units (photon/ $/ \mathrm{sec} / \mathrm{cm}^{2} /$ steradian). DsRed2 expression was also observed using a fluorescent microscope (Leica Microsystems, Tokyo, Japan) under a 560-nm excitation light.

\section{Semi-quantitative RT-PCR}

After removal, liver samples were immediately frozen in liquid nitrogen and stored at $-80^{\circ} \mathrm{C}$ until analyzed. Total RNA was isolated from liver tissue with TRIzol LS Reagent (Invitrogen, CA, USA). To eliminate genomic DNA, extracted RNA was incubated with $1 \mathrm{U} / \mu 1$ of DNaseI at room temperature for $15 \mathrm{~min}, 25 \mathrm{mM}$ ethylenediaminetetraacetic acid was added to stop the reaction, and RNA was then incubated at $65^{\circ} \mathrm{C}$ for $10 \mathrm{~min}$. Hepatic RNA was incubated with oligo d (T) primers (Invitrogen, CA, USA) and dNTP mix at $70^{\circ} \mathrm{C}$ for 10 min, and then reverse transcribed with $200 \mathrm{U} / \mu \mathrm{l}$ of Super Script (Invitrogen, CA, USA) at $50^{\circ} \mathrm{C}$ for $90 \mathrm{~min}$ and $70^{\circ} \mathrm{C}$ for $90 \mathrm{~min}$. PCR was conducted using specific primer sets. For rat Cyp2c11: upstream 5'-CTG GTC CAA CAC CTC TCC CA-3' and downstream 5'-GTC CGA AAA GTC GAG GGG TA -3'; for rat Cyp2c12: upstream 5'-CTT GCC CCAAAT GGT TTG TTG-3' and downstream 5'-GAG TCT TGC ATA ACT CAA AT-3'; and for Glyceraldehyde-3-phosphate dehydrogenase (Gapdh): upstream 5'-TTC AAC GGC ACA GTC AAG3 ' and downstream 5'-TAC AAA CAC TAC CCA CAA$3^{\prime}$.

PCR for rat Cyp $2 c 11$ was run for 30 cycles $\left(95^{\circ} \mathrm{C}\right.$ for $30 \mathrm{~s}, 58^{\circ} \mathrm{C}$ for $50 \mathrm{~s}$, and $72^{\circ} \mathrm{C}$ for $30 \mathrm{~s}$ ), and PCR for
Cyp 2 c 12 was run for 32 cycles $\left(95^{\circ} \mathrm{C}\right.$ for $30 \mathrm{~s}, 54^{\circ} \mathrm{C}$ for $30 \mathrm{~s}$, and $72^{\circ} \mathrm{C}$ for $30 \mathrm{~s}$ ) using Takara rTaq (Takara, Shiga, Japan). PCR products were analyzed on a $1 \%$ agarose gel containing ethidium bromide with $1 \times \mathrm{TAE}$ buffer. mRNA signals were visualized and signal intensity was quantified using Image $\mathrm{J}$ software (NIH, USA). mRNA signals were then normalized to Gapdh mRNA, which was used as an internal control.

\section{Histology}

Serial sections (40- $\mu \mathrm{m}$ thick) that encompassed the preoptic areas were prepared using a freezing microtome. Sections were mounted on gelatin-coated glass slides (Wako), stained with cresyl violet (MERCK, Darmstadt, German), dehydrated, and cover slipped with ENTELLAN New (MERCK, Darmstadt, German). Images were acquired using a fluorescent microscope (BZ-9000, KEYENCE).

\section{Statistical analysis}

Group results are given as means \pm standard deviations. The Ekuseru-Toukei 2010 (Social Survey Research Information Co., Ltd., Tokyo, Japan) was used the statistical analysis. Statistical comparisons between groups were made by one-way ANOVA, followed by a BonferroniDunn test. A $P$-value of $<0.05$ was considered significant.

\section{Results}

\section{Hepatic Cyp mRNA expression profiles}

Relative male-specific Cyp2c11 mRNA expression levels in the liver in OVX+TP females were significantly increased as compared with those in sham female rats and were comparable to those in sham male rats (Fig. 1 (a)). Relative female-specific Cyp2c12 mRNA expression levels in the liver were lower in OVX+TP females than in sham females, although this difference was not significant (Fig. 1 (b)). There were no significant differences in the expressions of these two mRNAs between OVX+TP females and sham males. There were also no significant differences in the expressions of these two mRNAs between OVX females, TP females, and sham female rats.

Cast male rats had significantly lower Cyp2c11 mRNA expression levels and significantly higher Cyp2c12 mRNA levels than those in sham male rats. Cast male rats had Cyp mRNA expression patterns similar to those of females. 
(a)

(b)
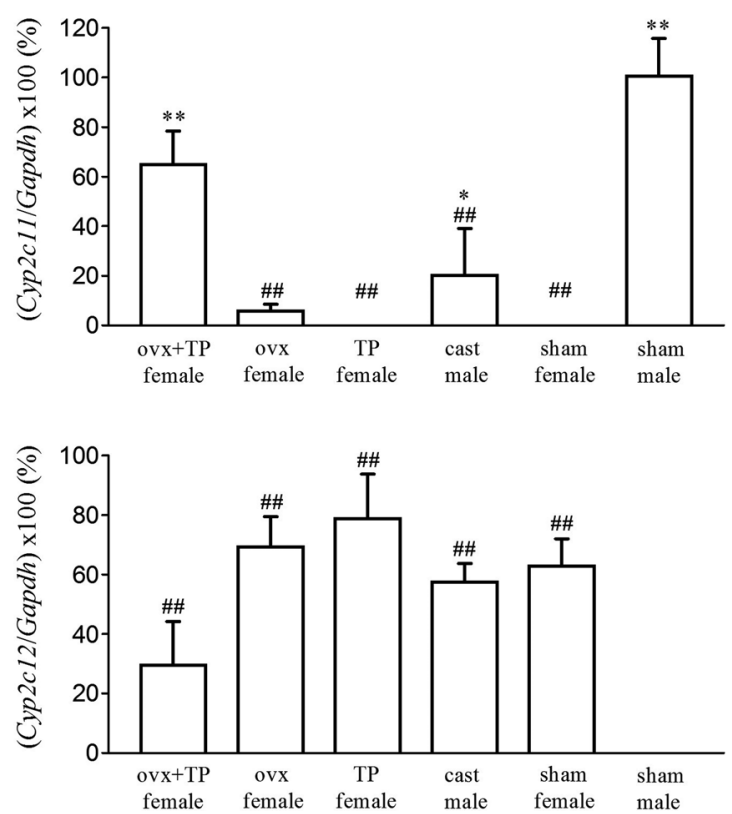

Fig. 1. Quantifying hepatic Cyp mRNA expression using semiquantitative PCR. Hepatic Cyp mRNA expression was determined when rats were 9-weeks old. (a): Male-specific Cyp 2c11 mRNA expression levels normalized to Gapdh mRNA used as an internal control. (b): Female-specific Cyp2c12 expression levels normalized to Gapdh mRNA. Results are means \pm standard deviations $(\mathrm{n}=4)$ (\#\#; vs. sham male, $P<0.01$, **; vs. sham female, $P<0.01$, *; vs. sham female, $P<0.05$ ).

\section{Hepatic DsRed2 expression profiles}

Neonatal OVX+TP females exhibited a high hepatic DsRed2 expression level, which was not detected among sham females (Fig. 2 (a)). Cast males did not express hepatic DsRed2 at levels that were close to the levels of sham males. Hepatic DsRed2 expression levels in OVX females and TP females were not significantly different from those in sham females.

The mean hepatic DsRed2 fluorescence expression level in OVX+TP females was $1.03 \times 10^{12} \pm 1.18 \times 10^{12}$ (Fig. 2 (b)), which was higher than the mean level in sham females $\left(1.43 \times 10^{10} \pm 1.78 \times 10^{9}\right)$; however, this difference was not significant. Cast male rats had reduced DsRed2 expression levels $\left(5.13 \times 10^{10} \pm 9.36 \times 10^{9}\right)$, but this level was not significantly different from that in sham females. OVX females and TP females had mean expression levels of $3.15 \times 10^{10} \pm 2.81 \times 10^{10}$ and 4.60 $\times 10^{10} \pm 3.30 \times 10^{10}$, respectively. These levels were comparable with that in sham females.
Correlations between Cyp mRNA and hepatic DsRed 2 expressions

We next assessed for a possible correlation between hepatic DsRed2 expression and Cyp mRNA expression. There was a positive correlation between DsRed2 expression and male-specific Cyp2c11 mRNA expression, and there was a negative correlation between DsRed2 expression and female-specific Cyp2c12 mRNA expression (Fig. 3). The $\mathrm{R}^{2}$ value for the DsRed2 and Cyp2c11 expression correlation result was 0.976 , and that for the DsRed2 and Cyp2c12 correlation result was 0.883 . DsRed2 expression was particularly strongly correlated with Cyp2c11 expression.

\section{SDN-POA volumes}

Sexually dimorphic nuclei of the preoptic area (SDNPOA) volumes for all rat groups are shown in Fig. 4. SDN-POA volumes were greater in male rats than in female rats. SDN-POA volumes were similar for TP females and sham males. Cast males had SDN-POA volumes that were comparable to those of sham females. OVX females had SDN-POA volumes similar to those of sham females.

\section{Discussion}

CYPs facilitate steroid and xenobiotic metabolism in the liver and are important for maintaining the body's homeostasis. These CYPs differ based on sex and animal species, and many types of CYPs that show sexually dimorphic expression have been identified $[2,4,11,12$, $14,15,23]$. These sex differences strongly depend on differences in GH-secretory patterns from the pituitary anterior lobe $[18,21]$. We previously established a transgenic (Alb-DsRed2 Tg) rat that expressed red fluorescent DsRed2 protein, particularly in hepatocytes, to visualize cell differentiation and multiplication and found that hepatic DsRed2 expression exhibited sexual dimorphism that was limited to adult males. The mechanism of the sexual dimorphism in DsRed2 expression is unclear. In this study, we examined the relevance of the sexually dimorphic expression patterns of GH-dependent Cyps and that of DsRed2 in Alb-DsRed2 Tg rats. Several sexually dimorphic CYPs are dependent on pituitary GH-secretory patterns, which are determined by sex steroid hormones during the postnatal period $[18,21]$. To reverse the GH secretory patterns in male and female $\mathrm{Tg}$ rats in adulthood, we experimentally reversed their 
(a)
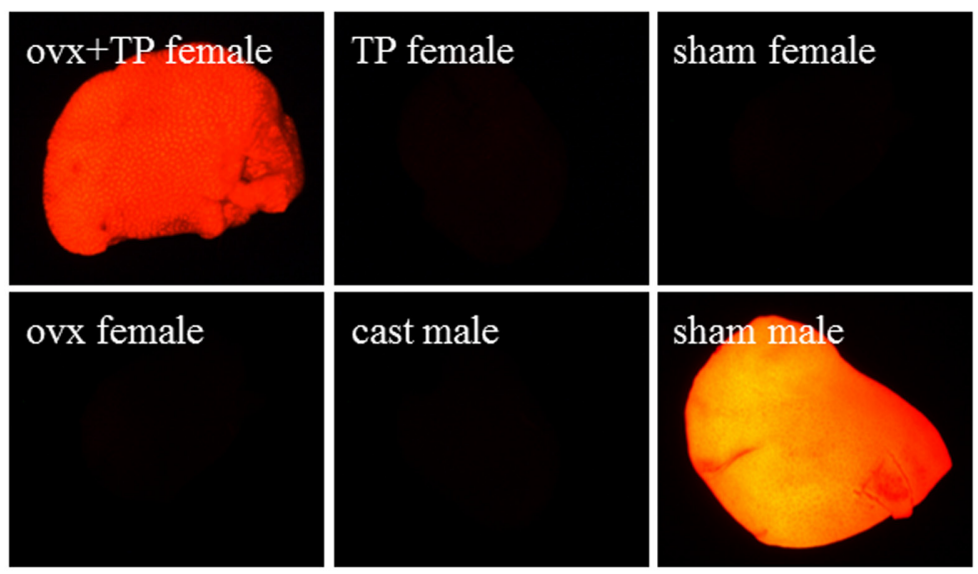

(b)

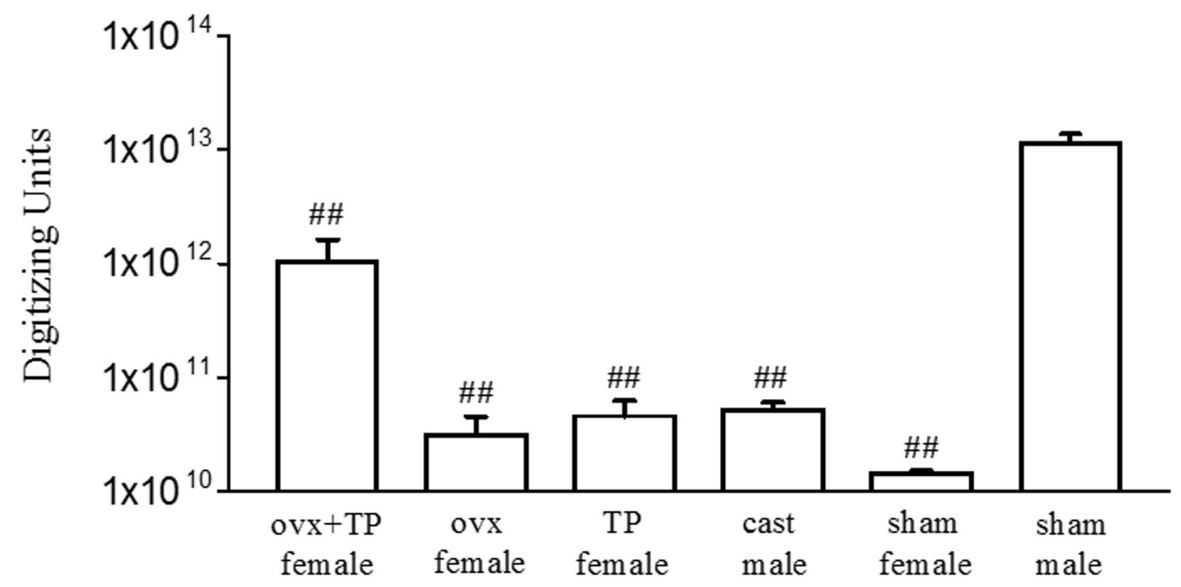

Fig. 2. Hepatic DsRed 2 expression in adult Tg rats. (a): Females that underwent neonatal ovariectomy and neonatal TP treatment (OVX+TP female), females that underwent neonatal ovariectomy only (OVX female), females that underwent neonatal TP treatment only (TP female), males that underwent neonatal castration (Cast male), sham-treated females (sham female), and sham-treated males (sham male). Each sample was observed by fluorescent microscopy when rats were 9-weeks old. (b): IVIS evaluation of DsRed2 expression levels in each group and quantified using analysis software. Results are means \pm standard deviations $(\mathrm{n}=4)$ (\#\#; vs. sham male, $P<0.01$ ).

sex hormone environments at the postnatal period using previously described methods [9].

We confirmed that OVX+TP females showed malespecific patterns of hepatic Cyp2c11 and DsRed2 expression; however, these levels were not comparable with the levels found in sham male rats. We considered that the GH-secretory patterns of the OVX+TP female rats did not completely mimic the male pattern because adult males secrete high concentrations of testosterone from their testes, whereas females obviously could not continuously produce testosterone.

The female-specific Cyp2c12 and DsRed2 expression levels in OVX females and TP females showed that ovariectomy did not affect Cyps and hepatic DeRed2 expression both in newborns and adults [1], and that TP treatment only could not induce male-specific gene expression in the liver. In a previous study, we found that castrating adult Tg males did not change either Cyp or 


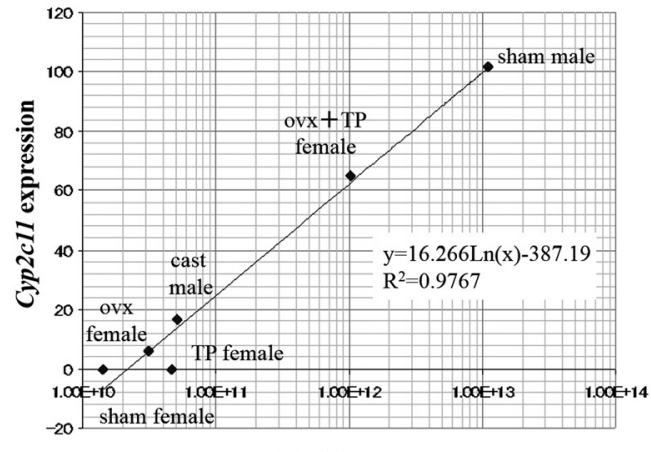

DsRed2 expression

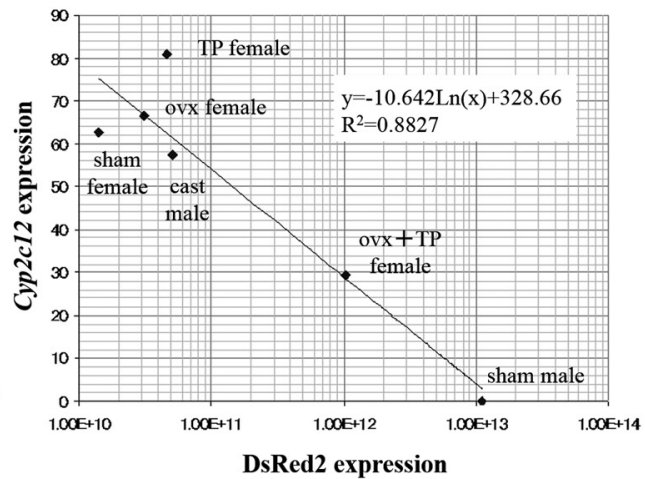

Fig. 3. Correlations between hepatic sexual dimorphic DsRed2 and Cyp expressions for all six groups of rats $(n=4)$.
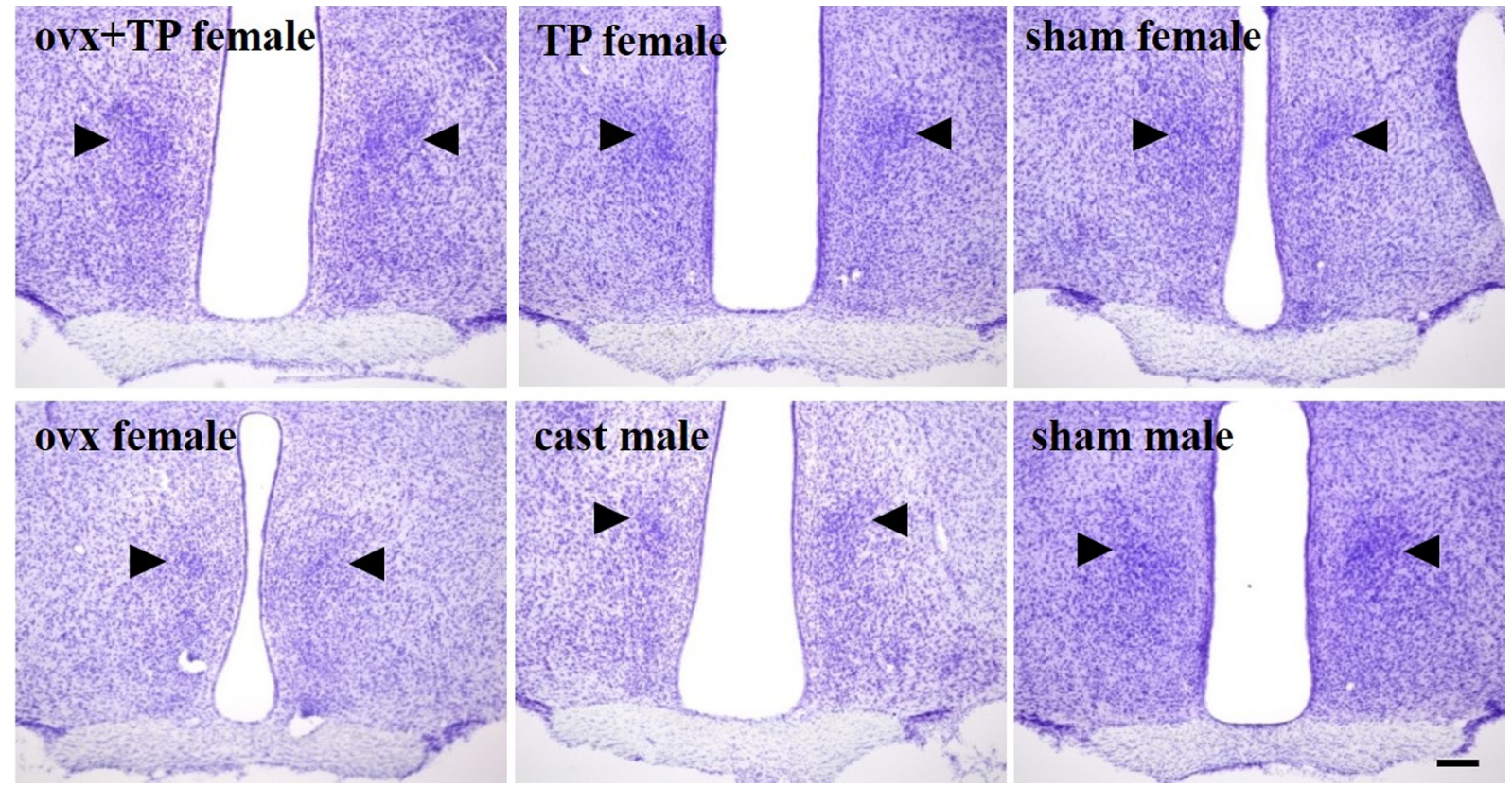

Fig. 4. Sexually dimorphic nuclei of the preoptic area (SDN-POA) volumes in 9-week-old rats. SDN-POA volumes in OVX+TP female rats, OVX female rats, TP female rats, Cast male rats, sham female rats, and sham male rats $(\mathrm{n}=4)$. Arrows represent SDN-POA region. Scale bar $=200 \mu \mathrm{m}$.

DsRed2 expression [1]. These results were supported by a report that $\mathrm{GH}$ secretion in adult rats was altered after neonatal gonadectomy but not after prepubertal gonadectomy [8], which suggested that only neonatal castration could change the GH secretory pattern, and thus, GH-dependent gene expression was also altered in Cast males.

We found that DsRed2 expression was positively correlated with male-specific Cyp2c11 expression and negatively correlated with female-specific Cyp $2 c 12$ expression, which revealed a strong correlation between the expression of GH-dependent Cyps and DsRed2. This strongly suggested that sexual dimorphic DsRed2 expression in these Tg rats was regulated by the GH secretory pattern, which is determined during the postnatal period. However, the mechanisms for why a mouse albumin promoter with a $\mathrm{Tg}$ rat induces GH-dependent sexual dimorphism in rat hepatocytes remain unclear. Sexual dimorphism of hepatic DsRed2 expression in this $\mathrm{Tg}$ rats was also observed in another sub-line. Furthermore, transgenic rats produced by connecting another target gene to the same mouse albumin promoter showed same differences which the introduced gene was strongly expressed in adult males but not in females [20]. 
Therefore, we predict that the cause of the sexual dimorphism of this DsRed2 expression depends on the regulation of the promoter but not gene insertion mutation. Interestingly, transgenic mice with this mouse albumin promotor did not show any sex differences [16], and it remains to be unexplained the cause of GH-dependent sexual dimorphism in rats. It is necessary to examine the detailed property of this promoter.

In this study, we did not directly assess plasma GH secretory patterns. However, we confirmed SDN-POA, a classic marker of sexual differentiation of the rat brain, which is involved in regulating rat sexual behavior [3]. SDN-POA volumes are considerably greater in males than in females and can be clearly identified by Nissl staining. Consistent with some classic experiments, we showed that cerebral sexual differentiation could be reversed in male and female rats by reversing the sex hormone environment during the perinatal period, which also established the effect of this neonatal treatment. Together with the results of hepatic Cyps and DsRed2 expression profiles, we considered that this treatment during the postnatal period could reverse the GH secretory patterns in male and female rats.

It is known that exposure to sex steroid hormones during pregnancy and the perinatal period has a significant effect on the growth and physiology of the fetus. As a cause for sex differences in the efficacy and adverse effects of drugs in men and women, sexual dimorphism of CYPs has become an important clinical problem, and numerous studies on this problem have been performed. However, the molecular mechanisms that contribute to these sex differences in CYPs are less well established because of the difficulty with continuously monitoring GH secretion patterns. Monitoring GH levels requires repetitive atrial blood sampling using a catheter [22] or mimicking plasma GH patterns in vitro using cultured hepatocytes from hypophysectomized rats without endogenous GH [19]. This in vivo bio-imaging system using DsRed2 expression as an indicator may be quite beneficial for monitoring changes in $\mathrm{GH}$ secretion. We would like to emphasize that this model is a completely new approach for evaluating GH secretion patterns and GH-dependent gene expression.

Our results demonstrated that GH-dependent endogenous Cyps expression and DsRed2 expression in this Tg rat model were strongly correlated. Thus, this Tg rat model can be a beneficial analytical tool for noninvasively and simply monitoring changes in Cyps expression using a fluorescent protein and also for research on the mechanisms for establishing sexual dimorphism.

\section{Acknowledgments}

We thank all staff members in the Department of Basic Science, School of Veterinary Nursing and Technology, Nippon Veterinary and Life Science University. Grants for this research were from the Japanese Ministry of Education, Science, Sports, and Culture (Basic Research [C], \# 24500498, to YH).

\section{References}

1. Arao, Y., Hakamata, Y., Igarashi, Y., Sato, Y., Kayama, F., Takahashi, M., Kobayashi, E., and Murakami, T. 2009. Characterization of hepatic sexual dimorphism in Alb-DsRed2 transgenic rats. Biochem. Biophys. Res. Commun. 382: 4650. [Medline] [CrossRef]

2. Cheung, C., Yu, A.M., Chen, C.S., Krausz, K.W., Byrd, L.G., Feigenbaum, L., Edwards, R.J., Waxman, D.J., and Gonzalez, F.J. 2006. Growth hormone determines sexual dimorphism of hepatic cytochrome P450 3A4 expression in transgenic mice. J. Pharmacol. Exp. Ther. 316: 1328-1334. [Medline] [CrossRef]

3. Gorski, R.A., Gordon, J.H., Shryne, J.E., and Southam, A.M. 1978. Evidence for a morphological sex difference within the medial preoptic area of the rat brain. Brain Res. 148: 333-346. [Medline] [CrossRef]

4. Guengerich, F.P. 1999. Cytochrome P-450 3A4: regulation and role in drug metabolism. Annu. Rev. Pharmacol. Toxicol. 39: 1-17. [Medline] [CrossRef]

5. Hakamata, Y., Tahara, K., Uchida, H., Sakuma, Y., Nakamura, M., Kume, A., Murakami, T., Takahashi, M., Takahashi, R., Hirabayashi, M., Ueda, M., Miyoshi, I., Kasai, N., and Kobayashi, E. 2001. Green fluorescent protein-transgenic rat: a tool for organ transplantation research. Biochem. Biophys. Res. Commun. 286: 779-785. [Medline] [CrossRef]

6. Inoue, H., Murakami, T., Ajiki, T., Hara, M., Hoshino, Y., and Kobayashi, E. 2008. Bioimaging assessment and effect of skin wound healing using bone-marrow-derived mesenchymal stromal cells with the artificial dermis in diabetic rats. J. Biomed. Opt. 13: 064036. [Medline] [CrossRef]

7. Inoue, H., Ohsawa, I., Murakami, T., Kimura, A., Hakamata, Y., Sato, Y., Kaneko, T., Takahashi, M., Okada, T., Ozawa, K., Francis, J., Leone, P., and Kobayashi, E. 2005. Development of new inbred transgenic strains of rats with LacZ or GFP. Biochem. Biophys. Res. Commun. 329: 288-295. [Medline] [CrossRef]

8. Jansson, J.O., Ekberg, S., Isaksson, O.G., and Edén, S. 1984. Influence of gonadal steroids on age- and sex-related secretory patterns of growth hormone in the rat. Endocrinology 114: 1287-1294. [Medline] [CrossRef]

9. Jansson, J.O. and Frohman, L.A. 1987. Differential effects of neonatal and adult androgen exposure on the growth hormone secretory pattern in male rats. Endocrinology 120 : 
1551-1557. [Medline] [CrossRef]

10. Liao, D.J., Blanck, A., Eneroth, P., Gustafsson, J.A., and Hällström, I.P. 2001. Diethylnitrosamine causes pituitary damage, disturbs hormone levels, and reduces sexual dimorphism of certain liver functions in the rat. Environ. Health Perspect. 109: 943-947. [Medline] [CrossRef]

11. MacGeoch, C., Morgan, E.T., Halpert, J., and Gustafsson, J.A. 1984. Purification, characterization, and pituitary regulation of the sex-specific cytochrome P-450 15 beta-hydroxylase from liver microsomes of untreated female rats. J. Biol. Chem. 259: 15433-15439. [Medline]

12. Morgan, E.T., MacGeoch, C., and Gustafsson, J.A. 1985. Hormonal and developmental regulation of expression of the hepatic microsomal steroid 16 alpha-hydroxylase cytochrome P-450 apoprotein in the rat. J. Biol. Chem. 260: 11895-11898. [Medline]

13. Murakami, T. and Kobayashi, E. 2005. Color-engineered rats and luminescent LacZ imaging: a new platform to visualize biological processes. J. Biomed. Opt. 10: 41204. [Medline] [CrossRef]

14. Noshiro, M., Serabjit-Singh, C.J., Bend, J.R., and Negishi, M. 1986. Female-predominant expression of testosterone 16 alpha-hydroxylase ("I"-P-450(16)alpha) and its repression in strain 129/J. Arch. Biochem. Biophys. 244: 857-864. [Medline] [CrossRef]

15. Pampori, N.A. and Shapiro, B.H. 1996. Feminization of hepatic cytochrome $\mathrm{P} 450$ s by nominal levels of growth hormone in the feminine plasma profile. Mol. Pharmacol. 50: 1148-1156. [Medline]

16. Saito, M., Iwawaki, T., Taya, C., Yonekawa, H., Noda, M., Inui, Y., Mekada, E., Kimata, Y., Tsuru, A., and Kohno, K. 2001. Diphtheria toxin receptor-mediated conditional and targeted cell ablation in transgenic mice. Nat. Biotechnol.
19: 746-750. [Medline] [CrossRef]

17. Sato, Y., Igarashi, Y., Hakamata, Y., Murakami, T., Kaneko, T., Takahashi, M., Seo, N., and Kobayashi, E. 2003. Establishment of Alb-DsRed2 transgenic rat for liver regeneration research. Biochem. Biophys. Res. Commun. 311: 478-481. [Medline] [CrossRef]

18. Shapiro, B.H., Agrawal, A.K., and Pampori, N.A. 1995. Gender differences in drug metabolism regulated by growth hormone. Int. J. Biochem. Cell Biol. 27: 9-20. [Medline] [CrossRef]

19. Thangavel, C. and Shapiro, B.H. 2007. A molecular basis for the sexually dimorphic response to growth hormone. Endocrinology 148: 2894-2903. [Medline] [CrossRef]

20. Ueda, M., Ando, Y., Hakamata, Y., Nakamura, M., Yamashita, T., Obayashi, K., Himeno, S., Inoue, S., Sato, Y., Kaneko, T., Takamune, N., Misumi, S., Shoji, S., Uchino, M., and Kobayashi, E. 2007. A transgenic rat with the human ATTR V30M: a novel tool for analyses of ATTR metabolisms. Biochem. Biophys. Res. Commun. 352: 299-304. [Medline] [CrossRef]

21. Waxman, D.J. and Holloway, M.G. 2009. Sex differences in the expression of hepatic drug metabolizing enzymes. Mol. Pharmacol. 76: 215-228. [Medline] [CrossRef]

22. Waxman, D.J., Pampori, N.A., Ram, P.A., Agrawal, A.K., and Shapiro, B.H. 1991. Interpulse interval in circulating growth hormone patterns regulates sexually dimorphic expression of hepatic cytochrome P450. Proc. Natl. Acad. Sci. USA 88: 6868-6872. [Medline] [CrossRef]

23. Wong, G., Kawajiri, K., and Negishi, M. 1987. Gene family of male-specific testosterone 16 alpha-hydroxylase (CP-450(16) alpha) in mouse liver: cDNA sequences, neonatal imprinting, and reversible regulation by androgen. Biochemistry 26: 8683-8690. [Medline] [CrossRef] 